\title{
ON LALR(k) TESTING
}

\author{
Seppo Sippu ${ }^{\dagger}$ and E1jas Soisalon-Soininen \\ Department of Computer Science \\ University of Helsinki \\ Tukholmankatu 2 \\ SF-00250 Helsinki 25, Finland
}

Abstract. The problem of testing whether or not a context-free gramar possesses the LALR(k) property is studied. The uniform problem (i.e. both the subject grammar and the integer $\mathrm{k}$ are problem parameters) is shown to be complete for polynomial space (PSPACE) when $k$ is expressed in unary, and complete for nondeterministic (one-level) exponential time (NE) when $\mathrm{k}$ is expressed in binary. This solves an open problem by Hunt, Szymanski and 011man, who showed that for $k$ in binary $L R(k), L L(k)$ and even strong LL(k) testing is NE-complete, and that LALR(k) testing is at least NE-hard. For $k$ in unary the lower bound of the problem follows from the recently obtained result that even for fixed $k \geqslant 1$ (i.e. only the subject grammar is a problem parameter) the problem is PSPACE-hard. Thus, the results lead to the striking conclusion that for $\mathrm{k}$ in binary LALR(k) testing is no harder (with respect to polynomial time reductions) than e.g. strong $L L(k)$ testing, and for $k$ in unary no harder than LALR(1) testing.

\section{INTRODUCTION}

Hunt, Szymanski and Ullman [3] study the problem of determining whether or not a context-free gramar is a member of some easily parsed subclass of grammars, such as the LR(k) grammars, SLR $(k)$ grammars, LALR(k) grammars, LL $(k)$ grammars and strong LL(k) grammars. For each fixed value of the integer $k$ they give an efficient polynomial time algorithm for the problem except when the class of grammars is the LALR( $k)$ grammars. For LALR(k) grammars the problem has recently been shown to be PSPACE-complete [7].

For the uniform problem (i.e. both the grammar and the integer $k$ are problem parameters) Hunt, Szymanski and V1lman [3] show that the running time of any algorithm solving the problem depends strongly on the representation chosen for $\mathrm{k}$. If $\mathrm{k}$ is expressed in unary, uniform $L R(k)$ testing as well as uniform SLR(k), LL(k) and strong $\mathrm{LL}(\mathrm{k})$ testing is NP-complete, and for $\mathrm{k}$ expressed in binary these testing problems are complete for nondeterministic one-level exponential time (NE). For uniform LALR(k) testing Hunt, Szymanski and Ullman only derive that the problem is at least $\mathrm{NP}$-hard for $\mathrm{k}$ in unary and at least $\mathrm{NE}$-hard for $\mathrm{k}$ in binary, and, on the other

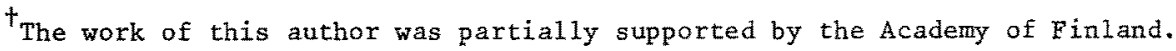


hand, they note that no better upper bound than the one obtained from the usual LALR(k) parser construction algorithm is known. This bound is two-level exponential when $k$ is expressed in binary.

In this paper we give tight bounds for the problem of uniform LALR( $k$ ) testing by showing that the problem is PSPACE-complete when $\mathrm{k}$ is expressed in unary and NEcomplete when $\mathrm{k}$ is expressed in binary. The latter result is especially interesting in that it shows how the complexity of uniform testing problems is dominated by $\mathrm{k}$. For any fixed $k$ the problem of whether or not a gramar possesses e.g. the strong $L L(k)$ property can be solved in deterministic time $O\left(n^{k+1}\right)$ where $n$ is the size of the grammar in question [6], whereas even LALR(1) testing is PSPACE-complete [7]. However, the corresponding uniform problems when $k$ has the "natural" binary representation are both of the same complexity.

\section{BACKGROUND}

We make free use of the notations and definitions given in [1] concerning strings and (context-free) grammars. We recall the convention that ( 1 ) A,B, and $\mathrm{C}$ denote nonterminals, (2) $\mathrm{a}, \mathrm{b}$, and $\mathrm{c}$ denote terminals, (3) $\mathrm{X}, \mathrm{Y}$, and $\mathrm{Z}$ denote either nonterminals or terminals, (4) terminal strings are represented by $u, v, \ldots, z$, whereas general strings are represented by $\alpha, \beta, \ldots, \omega$, and (5) the empty string is denoted by $\varepsilon$. As usual, we assume that every nonterminal and terminal can be used in the derivation of some sentence in the language. For convenience, we assume that all grammars are augmented in that there is a special "start production" $S^{\prime} \rightarrow S \$$ in which $S^{\prime}$ is the start symbol of the grammar and $\$$ is a special terminal (neither $S$ ' nor \$ may appear elsewhere in the grammar).

By the size $|G|$ of gramar $G$ we mean the sum of the lengths of the productions in $G$ (the length of production $A \rightarrow \omega$ is the length $|A \omega|$ of the string $A \omega)$. Clearly, $G$ can be encoded as a binary string of length $O(|G| \log |\Sigma|)$, where $\Sigma$ is the terminal vocabulary of $G$. For simplicity, we follow the convention of [3] and use |G| instead of $|G| \log |\Sigma|$ (or $|G| \log |G|$ ) as the measure of the size of the input for our gramar testing algorithms.

Pair $[A \rightarrow \alpha, \beta, y]$ is a $k$-item of $G$ (for integer $k \geqslant 0$ ) if $A \rightarrow \alpha \beta$ is a production of $\mathrm{G}$ and $\mathrm{y}$ is in $\operatorname{FIRST}_{\mathrm{k}}\left(\Sigma^{*}\right)$. The dotted production $A \rightarrow \alpha, \beta$ and the string $y$ are the core and the lookahead string, respectively, of the item.

String $Y$ of $G$ is a viable prefix of $G$ if

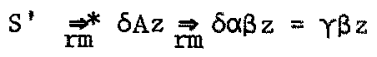

holds in $G$ for some string $\delta$ and terminal string $z$. Item $[A \rightarrow \alpha, \beta, y]$ is $L R(k)-v a] i d$ for $Y$ if (1) holds for some $\delta$ and $z$ such that $\operatorname{FIRST}_{k}(z)=y$.

Item $[A \rightarrow \alpha X . \beta, y]$ is the $X$-successor of item $[A \rightarrow \alpha . X \beta, y]$ if $X$ is a single symbol. Item $I^{\prime}=[B \rightarrow . \omega, z]$ is a direct $\underline{L R}(k)$-descendant of item $I=[A \rightarrow \alpha . B \beta, y]$, written $I D_{k} I^{\prime}$, if $z$ is in $\operatorname{FIRST}_{k}(\beta y)$. The closure of the set $\left\{\left[S^{\prime} \rightarrow . S \$, \varepsilon\right]\right\}$ under 
the descendant relation $D_{k}$ is called the initial state of the $L R(k)$-collection for $G$, and denoted by $q_{k}$. If $q$ is a set of $i$ tems and $X$ is a symbol of $G$, then $G_{0 T O}(q, X)$ denotes the closure under $D_{k}$ of the set $q^{\prime}$ consisting of the $X$-successors of items in $q$. The function $\mathrm{GOTO}_{k}$ is extended to general strings by the equations $\operatorname{GOTO}_{k}(q, \varepsilon)=q$ and $\operatorname{GOTO}_{k}(q, Y X)=$ GOTO $_{k}\left(\operatorname{GOTO}_{k}(q, \gamma), X\right)$. The set $\operatorname{GOTO}_{k}\left(q_{k}, \gamma\right)$ is non-empty if and only if $Y$ is a viable prefix of $G$; when non-empty, $\operatorname{GoTO}_{k}\left(q_{k}, Y\right)$ consists exactly of the $L R(k)$-valid items for $\gamma$. Non-empty sets $G_{0 T O}\left(q_{k}, \gamma\right)$ are called states of the $L R(k)-$ collection for $G$.

Distinct items $[A \rightarrow \alpha, \beta, y]$ and $[B \rightarrow \omega ., z]$ exhibit an $L R(k)$-conflict if $z$ is in FIRST $_{k}(\beta y)$. Gramar $G$ is a non-LALR(k) gramar if $G$ has viable prefixes $\gamma$ and $\delta$, and distinct items $I$ and $J$ such that $I$ and $J$ exhibit an $L R(k)$-conflict, I is $L R(k)$-valid for $Y, J$ is LR $(k)$-valid for $\delta$, and $\operatorname{GOTO}_{0}\left(q_{0}, Y\right)=\operatorname{GOTO}_{0}\left(q_{0}, \delta\right)$, where $q_{0}$ is the initial state of the LR( 0$)$-collection for $G$. If $G$ is not non-LALR $(k)$ it is LALR(k).

By the problem of uniform LALR(k) testing we mean a problem with instances of the form code $(G)$ \#code $(k)$ in which code $(G)$ is the natural encoding of a grammar $G$ as a binary string of length $O(|G| \log |G|)$, and code( $k)$ is the unary or binary representation for an integer $k$. Instance code $(G) \# \operatorname{code}(k)$ is a solution of the problem if and only if $G$ is an LALR(k) grammar. The complement of the problem of uniform LALR(k) testing is called the problem of uniform non-LALR(k) testing.

We assume the reader is familiar with the problem classes P, NP, and PSPACE. A problem is in NE if it is solvable by a nondeterministic one-level exponential time bounded algorithm, i.e. by an $0\left(2^{\mathrm{p}(n)}\right)$ algorithm where $p(n)$ is a polynomial of $n$. Problem $\Pi^{\prime}$ is hard for class $C$ of problems ( $C$-hard, for short) if every problem in $C$ is polynomial time reducible to $\Pi^{\prime}$, i.e. for every problem $\Pi$ in $C$ there is a deterministic polynomial time bounded algorithm mapping each instance $I$ of $\pi$ to an instance $I^{\prime}$ of $\Pi^{\prime}$ such that $I$ is a solution of $\Pi$ if and only if $I^{\prime}$ is a solution of $\Pi^{\prime}$. Problem $\Pi^{\prime}$ is complete for $C$ (C-complete, for short) if it is in $C$ and is $C$-hard.

\section{THE COMPLEXITY OF UNIFORM LALR(k) TESTING}

The usual method for LALR(k) testing is that implied by the LALR(k) parser construction algorithm which involves constructing first the LR(0)-collection for the given gramnar and then adding lookahead to the items. Because a grammar G has exactly $|G|$ item cores and at most $|G|^{k}$ different lookahead strings of length $k$ or less, the size of the LALR(k) parser is $0\left(|G|^{k+1} \cdot 2^{|G|}\right)$. Thus, the space complexity of the algorithm is necessarily one-level exponential in $\mid$ code(G) \#code(k)| if code(k) is the unary representation for $k$, and two-level exponential if code( $k$ ) is the binary representation for $k$.

The efficient algorithms presented by Kunt, Szymanski and Ullman in [3] for LR(k) testing have been obtained by using succinct nondeterninistic representations for the LR(k)-collection. Note that the nondeterministic version of the $L R(k)$-collection in which the states are single $k$-iterns rather than sets of $k$-items is only of 
size $0\left(\mid \mathrm{G}^{2 k+2}\right)$. Using this representation for the LR(k)-collection it is possible to test a gramar $G$ for the $L R(k)$ property in deterministic time $O\left((k+1)^{3} \cdot|G|^{4 k+4}\right)$.

The main idea in $[3]$, where an $O\left((\mathrm{k}+1)^{3} \cdot|\mathrm{G}|^{\mathrm{k}+2}\right)$ deterministic time bound for LR $(k)$ testing is established, is to use a representation consisting of several nondeterministic automata only of size $O\left((\mathrm{k}+1)^{2} \cdot|\mathrm{G}|\right)$. There is one automaton, denoted by $M(G, u)$, in the representation for each terminal string $u$ of length $k$ or less. Each automaton $M(G, u)$ characterizes the LR(k) property only for the particular lookahead string $u$, and is therefore only tested for LR(k)-conflicts caused by $u$. This arrangement allows a very economic nondeterministic algorithm for testing a gramar $G$ for the non-LR(k) property: it suffices to guess a lookahead string u, to construct the small automaton $M(G, u)$, and to check $M(G, u)$ for LR $(k)$-conflicts caused by $u$.

In what follows, we show that the idea of guessing, a priori, the conflicting lookahead string u can also be used to obtain an economic nondeterministic algorithm for non-LALR(k) testing. For this purpose, we first modify the concepts of k-item and LR(k)-validity so that they fit the "u-approach".

If $\alpha$ is a general string and $u$ is a terminal string, we denote by FIRST $(\alpha)$ the set of ali suffixes $z$ of $u$ such that $z$ is a prefix of some terminal string derivable from $\alpha$. Item $[A \rightarrow \alpha, \beta, y]$ is a $u$-item if $y$ is a suffix of $u$. Item $[A \rightarrow \alpha, \beta, y]$ is LR(u)-valid for viable prefix $Y$ if the conditions

$$
S^{\prime} \underset{\mathrm{rm}}{\Rightarrow} \delta A z \underset{\mathrm{rn}}{\Rightarrow} \delta \alpha \beta z=\Upsilon \beta z \text { and } y \in \operatorname{FIRST}_{\mathrm{u}}(z)
$$

hold for some $\delta$ and $z$ (cf. $[3,6]$ ).

The following lemma delineates the relationship between the $L R(k)-$ and LR(u)validity.

Lemma 1. The following conditions are logically equivalent for every viable prefix $Y$, production $A \rightarrow \alpha \beta$ and terminal string $u$.

(a) There is a terminal string $y$ such that $[A \rightarrow \alpha, \beta, y]$ is an $L R(u)-v a l i d$ item for $Y$ and $u$ is in FIRST $_{k}(\Sigma * \$) \cap$ FIRST $_{u}(\beta y)$.

(b) There is a terminal string $y^{\prime}$ such that $\left[A \rightarrow \alpha, \beta, y^{\prime}\right]$ is an LR( $k$ ) -valid item for $\gamma$ and $u$ is in FIRST $_{k}\left(B y^{\prime}\right)$.

Proof. The proof is a straightforward application of the definitions of LR(k)and $\operatorname{LR}(\mathrm{u})$-validity. Note the role of the augmenting $\$$ : the condition $u \in$ FIRST $_{\mathrm{k}}\left(\Sigma^{*} \$\right.$ ) implies that $u$ either is exactly of length $k$ or ends with $\$$. $\square$

We say that $u$-item $[A \rightarrow \alpha X . \beta, y]$ is the LR( $u, X)$-successor of $u-i$ tem $[A \rightarrow \alpha . X \beta, y]$ if $X$ is a single symbol. Correspondingly, $u$-item $[B \rightarrow . \omega, z]$ is an $L R(u, \varepsilon)$-successor of u-item $[A \rightarrow \alpha, B \beta, y]$ if $z$ is in FIRST ${ }_{u}(\beta y)$. Sequence $I_{1} \ldots I_{n}$ of $u-i$ tems is an LR(u)-chain reading string $\alpha_{1} \ldots \alpha_{n}$ if $I_{1}$ is the initial item [S' $\left.\rightarrow . s \$, \varepsilon\right], \alpha_{1}=\varepsilon$, and $I_{i+1}$, for each $i=1, \ldots, n-1$, is an $\operatorname{LR}\left(u, \alpha_{i+1}\right)$-successor of $I_{i}$.

The LR(u)-chains constitute the nondeterministic version of what could be called the IR(u)-collection for $G$, i.e. the "u-counterpart" of the LR(k)-collection. The construction is essentially the same as in the nondeterministic finite automaton $M(G, u)$ presented in [3]. For convenience, we have used a formalism different from 
that in [3], because in our nondeterministic testing algorithm only two computations of the automaton, i.e. two LR(u)-chains, are considered at a time.

The following theorem delineates the correspondence between LR(u)-valid items for viable prefix $\gamma$ and $L R(u)$-chains reading $\gamma$.

Theorem 2. Let $G$ be a grammar and $u$ a terminal string. If $I_{1} \ldots I_{n}$ is an LR(u)chain reading string $\gamma$, then $\gamma$ is a viable prefix and $I_{n}$ is LR(u)-valid for $\gamma$. Conversely, if $Y$ is a viable prefix and $I$ an $L R(u)$-valid item for $Y$, then there is an $L R(u)$-chain $I_{1} \ldots I_{n}$ reading $\gamma$ and ending with $I_{n}=I$.

Proof. See the proof of Lemma 2.3 in [3]. Note that the proof is completely analogous to the corresponding proof of the correctness of the LR(k) construction, and follows from the similarity of the behavior of the lookahead operators FIRST $_{u}$ and FIRST $_{k}$, $\square$

The following lemma states that any LR(u)-valid item $I$ for viable prefix $\gamma$ can be reached by a short $L R(u)$-chain $I_{1} \ldots I_{n}$ such that the string $\gamma^{\prime}$ read by the chain $I_{1} \ldots I_{n}$ leads to the same state in the LR(0)-collection as $r$. This result comes into use when we establish an upper bound for the time complexity of our nondeterministic LALR(k) testing algorithm.

Lemma 3. Let $G$ be a grammar, $q_{0}$ the initial state of the LR(0)-collection for $G$, and $u$ a terminal string. If $I$ is an $L R(u)$-valid item for viable prefix $\gamma$, then there is a viable prefix $\gamma^{\prime}$ and an $L R(u)$-chain $I_{1} \ldots I_{n}$ reading $\gamma^{\prime}$ such that $I=I_{n}$, GOTO $_{0}\left(q_{0}, \gamma^{\prime}\right)=$ GOTO $_{0}\left(q_{0}, \gamma\right)$ and $n \leqslant(|u|+1) \cdot|G| \cdot 2^{|G|}$.

Proof. By Theorem 2, there is at least one viable prefix $\gamma^{\prime}$ (namely, $\gamma$ itself), and at least one $L R(u)$-chain $I_{1} \ldots I_{n}$ reading $\gamma^{\prime}$ and ending with $I$ such that $\operatorname{GOTO}_{0}\left(q_{0}, Y^{\prime}\right)=\operatorname{GOTO}_{0}\left(q_{0}, \gamma\right)$. Let $\gamma^{\prime}$ and $I_{1} \ldots I_{n}$ be such that the length $n$ of the chain $I_{1} \ldots I_{n}$ is minimal. We claim that $n \leqslant(|u|+1) \cdot|G| \cdot 2|G|$. For the sake of contradiction, we assume that $\mathrm{n}>(|\mathrm{u}|+1) \cdot|\mathrm{G}| \cdot 2^{|G|}$.

By the definition of $L R(u)$-chain, $\gamma^{\prime}$ is of the form $\alpha_{1} \ldots \alpha_{n}$ so that $I_{1} \ldots I_{1}$, for each $i$, reads $\alpha_{1} \ldots \alpha_{i}$ (here each $\alpha_{i}$ is either a single symbol or empty). The number of different items $I_{i}, I \leqslant i \leqslant n$, is bounded by $(|u|+1) \cdot|G|$, because $G$ has exactly $|G|$ item cores, and $u$ has exactly $|u|+1$ suffixes. Similarly, the number of different states GOTO $_{0}\left(q_{0}, \alpha_{1} \ldots \alpha_{i}\right), 1 \leqslant \mathrm{i} \leqslant \mathrm{n}$, is bounded by $2|G|$. Thus the number of different pairs $\left(\mathrm{I}_{i}, \operatorname{GOTO}_{0}\left(\mathrm{q}_{0}, \alpha_{1} \ldots \alpha_{i}\right)\right)$ is bounded by $(|\mathrm{u}|+1) \cdot|\mathrm{G}| \cdot 2^{|G|}$. Since $\mathrm{n}$ was assumed to be greater than this upper bound, then, for some $1 \leqslant i<j \leqslant n, I_{i}=$ $I_{j}$ and $\operatorname{GOTO}_{0}\left(q_{0}, \alpha_{1} \ldots \alpha_{i}\right)=\operatorname{GOTO}_{0}\left(q_{0}, \alpha_{1} \ldots \alpha_{j}\right)$. Let us denote by $\gamma^{\prime \prime}$ the string $\alpha_{1} \ldots \alpha_{i} \alpha_{j+1} \ldots \alpha_{n}$. By the definition of $\operatorname{LR}(u)$-chain, the sequence $I_{1} \ldots I_{i} I_{j+1} \ldots I_{n}$ is then an $L R(u)$-chain reading $\gamma^{\prime \prime}$. By Theorem $2, \gamma^{\prime \prime}$ is a viable prefix. Moreover, by the definition of GOTO 0, GOTO $_{0}\left(q_{0}, \gamma^{\prime \prime}\right)=$ COTO$_{0}\left(q_{0}, \gamma^{\prime}\right)$. Thus, we can conclude that $I_{1} \ldots I_{i} I_{j+1} \ldots I_{n}$ is an $L R(u)$-chain reading viable prefix $\gamma^{\prime \prime}$ and ending with $I$ such that $\operatorname{GOTO}_{0}\left(q_{0}, Y^{\prime \prime}\right)=\operatorname{GOTO}_{0}\left(q_{0}, \gamma\right)$. But this is a contradiction, because $I_{1} \ldots I_{n}$ was assumed to be of minimal length. 
We say that distinct $u-i$ tems $[A \rightarrow \alpha, \beta, y]$ and $\left[B \rightarrow \omega_{.}, u\right]$ exhibit an $L R(u)-$ conflict if $u$ is in $\operatorname{FIRST}_{\mathrm{u}}(\beta \mathrm{y})$.

The following theorem states that the non-LALR(k) property of a grammar can be characterized by a pair of short $L R(u)$-chains ending with u-items exhibiting an $\operatorname{LR}(u)$ - conflict.

Theorem 4. Let $\mathrm{G}$ be a grammar, $\mathrm{q}_{0}$ the initial state of the LR(0)-collection for $G$, and $k$ a non-negative integer. Then $G$ is a non-LALR(k) grammar if and only if there is a terminal string $u$ in $\operatorname{FrRST}_{k}\left(\Sigma^{*} \$\right)$, an $L R(u)$-chain $I_{1} \ldots I_{m}$ reading string $\gamma$ and an $L R(u)$-chain $J_{1} \ldots J_{n}$ reading string $\delta$ such that $I_{m}$ and $J_{n}$ exhibit an $L R(u)$-conflict, GOTO $_{0}\left(q_{0}, \gamma\right)=\operatorname{COTO}_{0}\left(q_{0}, \delta\right)$ and $m, n \leqslant(k+1) \cdot|G| \cdot 2^{|G|}$.

Proof. We first assume that $G$ is non-LALR(k), and prove that the condition of the theorem is satisfied. By the definition of the non-LALR(k) property, there are viable prefixes $\gamma_{1}$ and $\delta_{1}$, and two $L R(k)$-items $\left[A \rightarrow \alpha . \beta, y^{\prime}\right]$ and $[B \rightarrow \omega,, u]$ such that $\operatorname{coTo}_{0}\left(q_{0}, Y_{1}\right)=\operatorname{GOTO}_{0}\left(q_{0}, \delta_{1}\right),\left[A \rightarrow \alpha . \beta, y^{\prime}\right]$ is $L R(k)$-valid for $\gamma_{1},[B \rightarrow \omega ., u]$ is $L R(k)-$ valid for $\delta_{1}$, and $u$ is in $\operatorname{FIRST}_{k}\left(\beta y^{\prime}\right)$. By Lemma $1, u$ is in $\operatorname{FIRST}_{k}\left(\Sigma^{*} \$\right)$, and there is a terminal string $y$ such that $[A \rightarrow \alpha . \beta, y]$ is $\operatorname{LR}(u)$-valid for $\gamma_{1}$ and $u$ is in $\operatorname{FIRST}_{u}(\beta y)$. Similarly, $\left[B \rightarrow \omega_{,}, u\right]$ is $L R(u)$-valid for $\delta_{1}$. By Lema 3 , there are viable prefixes $\gamma$ and $\delta$, and $L R(u)$-chains $I_{1} \ldots I_{m}$ and $J_{1} \ldots J_{n}$ reading $\gamma$ and $\delta$, respectively, such that $I_{m}=[A \rightarrow \alpha, \beta, y], J_{n}=[B \rightarrow \omega ., u], \operatorname{GOTO}_{0}\left(q_{0}, Y\right)=\operatorname{GOTO}_{0}\left(q_{0}, \gamma_{I}\right), \operatorname{GOTO}_{0}\left(q_{0}, \delta\right)=$ $\operatorname{GOTO}_{0}\left(\mathrm{q}_{0}, \delta_{1}\right)$, m, $\mathrm{n} \leqslant(k+1) \cdot|G| \cdot 2^{|G|}$. In other words, the condition of the theorem is satisfied.

The converse implication is easier: if the condition of the theorem is satisfied, then, by Theorem 2, $\gamma$ and $\delta$ are viable prefixes, $I_{m}$ is LR(u)-valid for $\gamma$, and $J_{n}$ is LR(u)-valid for $\delta$. Furthermore, by Lemma 1 , there is a pair of $k$-items $I$ and $J$ exhibiting an $L R(k)$-conflict such that $I$ is $L R(k)$-valid for $\gamma$, and $J$ is $L R(k)$-valid for $\overline{0} . \square$

As an example, consider the grammar with productions $S \rightarrow a A a|A b| a B b \mid B a, A \rightarrow c$, $B \rightarrow C$, and with augmenting production $S^{\prime} \rightarrow S \$$. The grammar is a prototype of an LR(1) grammar which is not LALR(k) for any $k$. The non-LALR(1) property of the grammar follows, by Theorem 4, from the fact that there are $\operatorname{LR}(a)$-chains $\left[S^{\prime} \rightarrow . S \$, \varepsilon\right][S \rightarrow, a A a, \varepsilon]$ $[S \rightarrow a . A a, \varepsilon][A \rightarrow . c, a][A \rightarrow c ., a]$ and $\left[S^{\prime} \rightarrow . S \$, \varepsilon\right][S \rightarrow . B a, \varepsilon][B \rightarrow . c, a][B \rightarrow c ., a]$. The chains read viable prefixes ac and $c$, respectively, and end with items exhibiting an $L R(a)-$ conflict. Moreover, the state $\operatorname{GoTO}_{0}\left(q_{0}\right.$, ac) consists of the $i$ tems $[A \rightarrow c ., \varepsilon]$ and $\left[B \rightarrow c_{2}, \varepsilon\right]$, and equals the state $\operatorname{coTo}_{0}\left(q_{0}, c\right)$. $D$

For the purpose of establishing that uniform non-LALR(k) testing can be performed economically using the characterization of the non-LALR(k) property presented in Theorem 4, we need the following lemmas.

Lemma 5. For every grammar $G$ there is a grammar $G_{\mathrm{T}}$ of size $O(|G|)$ such that the following two conditions are satisfied.

(1) $G_{T}$ is in Chomsky normal form (e.g. [2]) except that it may contain productions of the form $A \rightarrow B$, where $B$ is a nonterminal. 
(2) For each production $A \rightarrow \alpha \beta$ in $G$ and non-empty strings $\alpha$ and $\beta$ there is a nonterminal $[\beta]$ in $G_{T}$ which generates exactly the non-empty sentences in the language $I(\beta)$.

Moreover, $G_{\mathrm{r}}$ can be constructed from $G$ in time $O(|\mathrm{G}|)$.

Proof. First, transform $G$ into two-form (e.g. [2]) by replacing each production $A \rightarrow X \beta$, where $\beta \neq \varepsilon$, by the production $A \rightarrow X[\beta]$, and by introducing for each new nonterminal $[X \beta]$ in which $\beta \neq \varepsilon$ the production $[X \beta] \rightarrow X[\beta]$, and for each new nonterminal $[X]$ the production $[X] \rightarrow X$. Then eliminate all $\varepsilon$-productions. If done carefully, this can be carried out in linear time (cf. [2]). Note that we camnot require $G_{\mathrm{T}}$ to be in true Chomsky normal form because the elimination of chain productions $A \rightarrow B$ may square the size of the grammar (cf. [2]).

Lemma 6. Let $G$ be a grammar, $G_{T}$ the transformed gramar of Lemma 5 , u a terminal string, I and $J$ u-items, and $\alpha^{\prime}$ a string of length $\left|\alpha^{*}\right| \leqslant 1$. Then it can be decided, in nondeterministic time $O((|u|+1) \cdot|G|)$, whether or not $J$ is an $L R\left(u, \alpha^{*}\right)-$ successor of $I$.

Proof. If $\alpha^{\prime} \neq \varepsilon$, then the task is trivial. As regards the case $\alpha^{*}=\varepsilon$, we use the fact that in an $\varepsilon$-free grammar, such as $G_{T}$, any sentence $v$ can be generated by using no more than $2 \mathrm{n} \mid \mathrm{v} / \mathrm{-n}$ derivation steps, where $\mathrm{n}$ is the number of nonterminals in the grammar. In other words, the generation is linear both in the length of the sentence and in the size of the grammar. If $I$ is of the form $[A \rightarrow \alpha . B \beta, y]$, where $\beta \neq$ $\varepsilon$, and if $J$ is of the form $[B \rightarrow . \omega, z]$, it thus suffices to nondeterministically generate from the nonterminal $[\beta]$ in $G_{T}$ a left sentential form $v \delta$, where $v$ is a terminal string, and then to check whether or not $z$ is a prefix of $v$, or $z$ is a prefix of vy and $\delta=\varepsilon \cdot \square$

Using a reasoning similar to that in the proof of Lemma 6 we obtain

Lemma 7. Let $G$ be a grammar, $G_{T}$ the transformed grammar of Lemma 5 , $u$ a terminal string, and $I$ and $J$ u-items. Then it can be decided, in nondeterministic time $O((|u|+1) \cdot|G|)$, whether or not I and J exhibit an LR(u)-conflict. $\square$

We can now state the main result of the present paper.

Theorem 8. Grammar $G$ can be uniformly tested for the non-LALR(k) property simultaneously in nondeterministic space $o(k+1) \cdot|G|)$ and in nondeterministic time $o\left((k+1)^{2} \cdot|G|^{2} \cdot 2^{|G|}\right)$

Proof. We outline a nondeterministic algorithm which uses the characterization of the non-LALR $(k)$ property presented in Theorem 4 . The idea in the algorithm is as follows. First, the algorithm guesses a lookahead string $u$ in FIRST $_{k}(\Sigma * \$)$. Then the algorithm generates, nondeterministically, a pair of (not necessarily distinct) $L R(u)$-chains $I_{1} \ldots I_{m}$ and $J_{1} \ldots J_{n}$. Along with the generation, the algorithm keeps track of the states $q_{i}=\operatorname{GOTO}_{0}\left(q_{0}, \alpha_{1} \ldots \alpha_{i}\right)$ and $r_{j}=\operatorname{coTO}_{0}\left(q_{0}, \beta_{1} \ldots \beta_{j}\right)$, where $q_{0}$ is the initial state of the $\operatorname{LR}(0)$-collection for $G$, and $\alpha_{1} \ldots \alpha_{j}$ and $\beta_{1} \ldots \beta_{j}$ are the strings read by the chains $I_{1} \ldots I_{i}$ and $J_{1} \ldots J_{j}$, respectively. The algorithm accepts its input whenever $I_{m}$ and $J_{n}$ exhibit an $L R(u)-\operatorname{conflict}$ and $q_{m}=r_{n}$. By Theorem 4, 
the algorithm accepts code(G)\#code(k) if and only if $G$ is a non-LALR(k) grammar.

Note that the algorithm can be made very economic in space: at any moment of the generation, only the current items $I_{i}$ and $J_{j}$, and states $q_{i}$ and $r_{j}$ need be present. The algorithm is also economic in time, because, by Theorem 4, there are always short LR(u)-chains leading to the acceptance of a non-LALR(k) grammar $G$.

The space and time bounds mentioned in the theorem are easily established. First, the guessing of the lookahead string $u$ takes at most time $o(k \cdot|G|)$, and the construction of the initial state $q_{0}$ of the LR(0)-collection for $G$ at most time $O(|G|)$. The generation of the $L R(u)$-chains begins with $I=\left[S^{\prime} \rightarrow . S \$, \varepsilon\right]=J$ as the current items, and $q=q_{0}=r$ as the current states. At any non-accepting situation, $i$.e. when $q \neq r$ or $I$ and $J$ do not exhibit an $L R(u)$-conflict, the algorithm guesses, in time $o((|u|+1) \cdot|G|)$, u-items $I_{1}$ and $J_{1}$, and strings $\alpha_{1}$ and $\beta_{1}$ of lengths $\left|\alpha_{1}\right| \leqslant 1$ and $\left|\beta_{1}\right| \leqslant 1$. Then the algorithm checks, nondeterministically, whether or not $I_{1}=I$ or $I_{1}$ is an $\operatorname{LR}\left(u, \alpha_{1}\right)$-successor of $I$, and whether or not $J_{1}=J$ or $J_{1}$ is an $\operatorname{LR}\left(u, \beta_{1}\right)-$ successor of $\mathrm{J}$. By Lemma 6 , this checking can be carried out in time $O((|u|+1) \cdot|\mathrm{G}|)$. In the affirmative case, the process is continued and new states $q_{1}$ and $r_{1}$ are generated. The state $q_{1}$ is eithex $q$ or GOTO $\left(q, \alpha_{1}\right)$, depending on whether $I_{1}$ was $I$ or an $L R\left(u, \alpha_{1}\right)$-successor of $I$. Similarly, the state $r_{1}$ is either $r$ or Goro $0_{0}\left(r, \beta_{1}\right)$. Clearly; $\mathrm{q}_{1}$ and $r_{1}$ can be constructed in time $0(|\mathrm{G}|)$. Then the algorithm checks whether or not $q_{1}=r_{1}$, and, nondeterministically, whether or not $I_{1}$ and $J_{1}$ exhibit an LR(u)-conflict. By Lemma 7 , this checking can be carried out in time $O((|u|+1) \cdot|\mathrm{G}|)$. In the affirmative case, the algorithm accepts its input. Otherwise, $I_{1}$ and $J_{1}$ are chosen as new current items $I$ and $J$, and $q_{I}$ and $r_{1}$ as new current states $q$ and $x$, after which the process is repeated.

If $G$ is a non-LALR(k) grammar, then, by Theorem 4 , there is a lookahead string $u$ of length at most $k$ and a pair of LR(u)-chains of length at most $(k+1) \cdot|G| \cdot 2|G|$ leading to the acceptance of $G$. Thus, we can conclude that the whole test for the non-LALR(k) property can be carried out simultaneously in nondeterministic space $O((k+1) \cdot|G|)$ and in nondeterministic time $O\left((k+1)^{2} \cdot|G|^{2} \cdot 2^{|G|}\right)$.

By Savitch's theorem (e.g. [2]) we have

Corollary 9. Grammar $G$ can be uniformly tested for the LALR(k) property in deterministic space $0\left((k+1)^{2} \cdot|G|^{2}\right)$. 口

By replacing the integer $k$ in the space bound of Corollary 9 by the length $|\mathrm{un}(\mathrm{k})| \mathrm{f}$ of the unary representation un( $k)$ for $k$, we get

Corollary 10. Uniform LALR(k) testing is in PSPACE when $k$ is expressed in unary. a

The problem of $\operatorname{LALR}(k)$ testing is shown in [7] to be PSPACE-hard even for fixed integers $k \geqslant 1$. The result is obtained by a reduction from the finite automaton nonuniversality problem. Since LALR(1) testing trivially reduces to uniform LALR(k) testing, we thus have, by Corollary 10 ,

Theorem 11. Uniform LALR(k) testing is PSPACE-complete when $k$ is expressed in unary $\square$ 
By replacing $k+1$ in the time bound of Theorem 8 by $2^{|b i n(k)|}$, in which bin(k) is the binary representation for $k$, we get

Corollary 12. Uniform non-LALR(k) testing is in NE when $k$ is expressed in binary. $\square$

Uniform non-LALR(k) (as well as non-LR(k), non-LL(k) etc.) testing is shown in [3] to be NE-hard. The result is obtained by the usual trick of constructing for given nondeterministic $T(n)$ time bounded Turing machine $M$ and input $x$ a gramar $G(M, x)$ which is ambiguous if and only if $M$ accepts $x$. For a sufficiently great $k$ (more specifically, $\left.k \geqslant 8 T(|x|)^{2}\right)$, the ambiguity of $G(M, x)$ is equivalent to the non-LALR(k), non-LL(k) etc. properties of $G(M, x)$.

Thus we have

Theorem 13. Uniform non-LALR(k) testing is NE-complete when $k$ is expressed in binary. $\square$

Theorem 8 and Corollary 9 also imply economic testing algorithms for fixed integers $k \geqslant 1$.

Corollary 14. Let $k \geqslant 1$ be a fixed integer. Then gramar $G$ can be tested for the LALR $(k)$ property in deterministic space $O\left(|G|^{2}\right)$, and for the non-LALR $(k)$ property in nondeterministic time $O\left(2^{|G|+2 \log |G|}\right)$. $\square$

\section{CONCLUSIONS}

In this section we combine the results of Section 3 with previously published results to categorize the complexity of different gramar problems. We consider the questions of whether or not a given context-free grammar is a member of the class of $\operatorname{LR}(k), \operatorname{SLR}(k), \operatorname{LALR}(k), \operatorname{LL}(k)$ or strong $L L(k)$ grammars both when the integer $k$ is fixed in advance and when $k$ is a parameter of the decision procedure. Basic results are given in Table 1 .

\begin{tabular}{|c|c|c|c|c|c|}
\hline $\begin{array}{l}\text { Grammar } \\
\text { class } \mathrm{C}(\mathrm{k}) \\
\text { Role of } \mathrm{k}\end{array}$ & $\begin{array}{c}\text { non-strong- } \\
L L(k)\end{array}$ & non-LL $(k)$ & non-SLR(k) & non-LR(k) & non-LALR (k) \\
\hline fixed $k \geqslant 1$ & in $P$ & in $P$ & in $\mathrm{P}$ & in $P$ & PSPACE-complete \\
\hline$k$ in unary & $\mathrm{NP}$-complete & $\mathrm{NP}$-complete & NP-complete & NP-complete & PSPACE-complete \\
\hline $\mathrm{k}$ in binary & NE-complete & NE-complete & $\mathrm{NE}$-complete & NE-complete & $\mathrm{NE}$-complete \\
\hline $\begin{array}{l}\text { existence of } \mathrm{k} \\
\text { such that } \mathrm{G} \text { is } \\
\text { in } \mathrm{C}(\mathrm{k})\end{array}$ & undecidable & undecidable & undecidable & undecidable & undecidable \\
\hline
\end{tabular}

Table 1. Complexity of the membership problem for some classes of grammars.

For fixed $k$ already Knuth [4] gave a polynomial time bounded algorithm for LR(k) testing. The best polynomial time bounded algorithms for SLR(k) and $L R(k)$ testing come from Hunt, Szymanski and Ullman [3], whereas the best time bounds for strong $\mathrm{LL}(\mathrm{k})$ and $\mathrm{LL}(\mathrm{k})$ testing come from the authors [6]. The result that LALR(k) testing 
for fixed $k \geqslant 1$ is PSPACE-complete is from [7]. For $k$ in unary as well as for $k$ in binary the results for LALR( $k$ ) testing are proved in Section 3, whereas for other classes of grammars the results are from [3]. The undecidability result for LR(k) grammars is proved by Knuth [4]; the proof can be easily modified for SLR( $k$ ) and LALR(k) grammars as well. The undecidability result for LL(k) and strong LI(k) grammars is from [5].

More detailed bounds for the complexity of the problems when $k \geqslant 1$ is a fixed integer are given in Table 2. The size $|G|$ of grammar $G$ is denoted by $n$.

\begin{tabular}{|c|c|c|c|c|c|}
\hline $\begin{array}{l}\text { Grammar } \\
\text { class } \mathrm{C}(\mathrm{k}) \\
\text { Resource }\end{array}$ & $\begin{array}{c}\text { non-strong- } \\
\text { LL }(\mathrm{k})\end{array}$ & non-LL $(k)$ & non-SLR $(k)$ & non-LR(k) & non-LALR $(k)$ \\
\hline $\begin{array}{l}\text { deterministic } \\
\text { time }\end{array}$ & $o\left(n^{k+1}\right)$ & $o\left(n^{k+1}\right)$ & $o\left(n^{k+2}\right)$ & $O\left(n^{k+2}\right)$ & $o\left(2^{n+(k+1) \log n}\right)$ \\
\hline $\begin{array}{l}\text { deterministic } \\
\text { space }\end{array}$ & $O(n)$ & $O(n)$ & $o\left(n^{2}\right)$ & $O\left(n^{2}\right)$ & $0\left(n^{2}\right)$ \\
\hline $\begin{array}{l}\text { nondetermin- } \\
\text { istic time }\end{array}$ & $O(n)$ & $O(n)$ & $O\left(n^{2}\right)$ & $O\left(n^{2}\right)$ & $O\left(2^{n+2} \log n\right)$ \\
\hline
\end{tabular}

Table 2. Complexity bounds for the membership problems when $k \geqslant 1$ is a fixed integer.

As we already noted the deterministic time bounds for $L L(k)$ and strong LL(k) testing are from [6]. The deterministic space bound and the nondeterministic time bound for these problems are implicit in [6]. All bounds for SLR(k) and LR(k) testing are from [3]. For LALR(k) testing the deterministic time bound is the one implied by the parser construction algorithm, whereas the deterministic space bound and the nondeterministic time bound are proved in Section 3 of the present paper. Observe that the deterministic time and space bounds are obtained simultaneously except for LALR(k) testing.

\section{REFERENCES}

1. Aho,A.V., and J.D.ullman, The Theory of Parsing, Translation and Compiling. Vol. 1: Parsing. Prentice-Hall, 1972.

2. Harrison,M.A., Introduction to Formal Language Theory. Addison-Wesley, 1978.

3. Hunt, H.B.,III, T.G.Szymanski and J.D.UlIman, On the Complexity of LR(k) Testing. Comm. ACM 18 (1975), 707-716.

4. Knuth,D.E., On the Translation of Languages from Left to Right. Information and Contro1 8 (1965), 607-639.

5. Rosenkrantz,D.J., and R.E.Stearns, Properties of Deterministic Top-Down Grammars. Information and Control 17 (1970), 226-256.

6. Sippu, S., and E.Soisalon-Soininen, Characterizations of the LL(k) Property. In: Automata, Languages and Programing, Seventh Colloquium, Noordwijkerhout, July 1980. Springer-Verlag, 1980, 596-608.

7. Ukkonen,E., and E,Soisalon-Soininen, LALR(k) Testing Is PSPACE-Complete. To be presented at the Thirteenth ACM Symposium on Theory of Computing, Milwaukee, May 1981. 